\title{
Immune Networks in Health and Disease
}

\author{
Heung Kyu Lee ${ }^{1}$ and Doo Hyun Chung ${ }^{2,3, *}$
}

${ }^{1}$ Graduate School of Medical Science and Engineering, Korea Advanced Institute of Science and Technology (KAIST), Daejeon 34141, Korea, '2Department of Pathology, Seoul National University College of Medicine, Seoul 03080, Korea, ${ }^{3}$ Laboratory of Immune Regulation, Department of Biomedical Sciences, Seoul National University College of Medicine, Seoul 03080, Korea

*Correspondence: doohyun@snu.ac.kr

https://doi.org/10.14348/molcells.2021.0117

www.molcells.org

The immune system comprises cells, chemicals, and processes that protect tissues and organs such as the skin, respiratory tract, digestive tract, and others exposed to antigens from external sources (Marshall et al., 2018). A variety of immune cells are involved in maintaining health but can sometimes exacerbate symptoms or induce autoimmune diseases. Many studies have been conducted to understand the immune system, which is generally divided into two categories: innate immunity and adaptive (or acquired) immunity (Chaplin, 2010). Immune regulation is a network of complex interactions between the cells responsible for innate and adaptive immunity. Understanding the tissue-specific roles of the immune cell network in innate and adaptive immunity is an essential step toward understanding diseases related to the immune system. To this end, we have discussed three issues of immune system such as innate and adaptive immunity, and immune regulation of tumor microenvironment.

In the first reviews for innate immunity, Je-Wook Yu and Doo Hyun Chung discuss macrophage-related processes essential for innate immunity. Macrophages are phagocytic cells and play a role in homeostasis, inflammation, protection, and tissue recovery (Mosser et al., 2021). Many macrophages are located strategically in organs where microbial invasion or accumulation of foreign substances occurs. Macrophages differentiate during development and are responsible for tissue-specific functions in each organ. Je-Wook Yu and colleagues review features unique to tissue-resident macrophages in the brain, while Doo Hyun Chung and colleagues highlight the development and function of alveolar macro- phages in the lungs.

Hye Young Kim and colleagues examine the more recently discovered innate lymphoid cells (ILCS). ILCS play a regulatory role in innate immunity (Vivier et al., 2018). Depending on the cell type, ILCs secrete interleukin (IL)-4, interferon $\gamma$, or IL17, respectively, and help regulate the tissue's appropriate immune response. Hye Young Kim and colleagues discuss the identified role of ILCS in various immune diseases and their relevance to treatment.

Innate immunity helps develop adaptive immunity, especially when innate immunity cannot effectively remove the causative agent of an infectious disease (Bonilla and Oettgen, 2010). Among T cells, CD1-restricted T cells play an important role in bridging innate and adaptive immunity. Ji Hyeong Kim and colleagues review group 1 CD1-restricted T cells in the adaptive immune response. In the classical $T$ cell activation pathway, the T cell receptor (TCR) signal transmitted through the major histocompatibility complex (MHC) molecule activates immune cells in a downstream pathway. However, in addition to MHC-mediated cell signaling, lipid antigens on CD1 molecules play an important role in the adaptive immune response (Vincent et al., 2003).

To understand adaptive immunity further, Eun Sook Hwang and colleagues discuss the differentiation of $\mathrm{T}$ helper (Th) cells (also known as CD4 T cells) during adaptive immunity. Th cells are divided into the subtypes Th1, Th2, Th17, and regulatory $T$ cells (Treg), which are generated under different conditions and induce various immune reactions (Luckheeram et al., 2012). Many reports show that abnormal

Received 11 May, 2021; accepted 12 May, 2021; published online 31 May, 2021 
differentiation and production of Th cell subtypes are associated with various autoimmune diseases. Transcription factors regulate Th cell differentiation, and Eun Sook Hwang and colleagues discuss additional post-translational modifications that affect Th cell differentiation.

The TCR itself has a low affinity to its counterpart of $\mathrm{MHC}$ molecules. Therefore, to effectively generate a strong interaction between $\mathrm{MHC}$ and $\mathrm{T}$ cell, $\mathrm{MHC}$ molecules take on oligomeric forms to create a stable connection with the corresponding T cell (Hadrup et al., 2009). Jun Chang discusses the latest findings on the interaction between $\mathrm{MHC}$ multimers and T cells.

Recent research highlights the importance of signaling substances and transcription factors for immune regulation. Zinc homeostasis plays a critical role in immune response signaling. After TCR stimulation, the concentration of zinc ions in the cytoplasm increases rapidly and influences the phosphorylation reaction regulation (Maywald et al., 2017). Won-Woo Lee and colleague discuss the role of zinc ions in immune-related signaling such as Toll-like receptors, TCR, and cytokine-mediated signal transduction.

Expanding on the signaling pathways of immune cells, You-Me Kim and colleagues discuss the role of the microphthalmia/transcription factor E (MiT/TFE) family of transcription factors in immunomodulation. The MiT/TFE family plays an important part in regulating autophagy and lysosomal biogenesis and has recently been shown to be an important regulator in cellular metabolic reprogramming (Yang et al., 2018). You-Me Kim and colleagues review the latest knowledge and mechanisms related to MiT/TFE proteins and describe novel, potential therapeutic strategies.

Recently, immune checkpoint blockade has been emerged as a successful therapeutic strategy for cancer patients. Heung Kyu Lee and colleague discuss the anti-tumor immune response. The immune response plays an important role in regulating tumors, and symbiotic microorganisms greatly influence these anti-tumor immune networks (lida et al., 2013). A change in intestinal microflora influences not only the effects of anti-tumor treatment but also their therapeutic toxicity. Heung Kyu Lee and colleague review how intestinal microbes affect the regulation of immune responses concerning tumors.

The immune network plays an important role in tumor control, and various attempts are being made to treat tumors by modulating these immune responses. However, these attempts can be problematic because responses vary depending on the patient or tumor type (Waldmann, 2003). Also, while the initial response to immunotherapy may be positive, acquired resistance to immunotherapy is a barrier. Sang-Jun $\mathrm{Ha}$ and colleagues examine various clinical perspectives to overcome acquired resistance to anti-PD-1/PD-L1 therapy, immunotherapy using a group of anti-cancer checkpoint inhibitors.

Immune system regulation is important for maintaining health and fighting various infectious diseases. However, an excessive immune response can induce detrimental effects like autoimmune diseases; therefore, the immune system requires precise control of the complex immune network. Past, current, and future research continue to reveal the complexity and nuances that balance the immune system as a whole and in tissue-specific locations.

\section{CONFLICT OF INTEREST}

The authors have no potential conflicts of interest to disclose.

\section{ORCID}

Heung Kyu Lee https://orcid.org/0000-0002-3977-1510

Doo Hyun Chung https://orcid.org/0000-0002-9948-8485

\section{REFERENCES}

Bonilla, F.A. and Oettgen, H.C. (2010). Adaptive immunity. J. Allergy Clin. Immunol. 125(2 Suppl 2), S33-S40.

Chaplin D.D. (2010). Overview of the immune response. J. Allergy Clin. Immunol. 125(2 Suppl 2), S3-S23.

Hadrup, S.R., Bakker, A.H., Shu, C.J., Andersen, R.S., van Veluw, J., Hombrink, P., Castermans, E., thor Straten, P., Blank, C., Haanen, J.B., et al. (2009). Parallel detection of antigen-specific T-cell responses by multidimensional encoding of MHC multimers. Nat. Methods 6, 520-526.

Iida, N., Dzutsev, A., Stewart, C.A., Smith, L., Bouladoux, N., Weingarten, R.A., Molina, D.A., Salcedo, R., Back, T., Cramer, S., et al. (2013). Commensal bacteria control cancer response to therapy by modulating the tumor microenvironment. Science 342, 967-970.

Luckheeram, R.V., Zhou, R., Verma, A.D., and Xia, B. (2012). CD4 ${ }^{+}$T cells: differentiation and functions. Clin. Dev. Immunol. 2012, 925135.

Marshall, J.S., Warrington, R., Watson, W., and Kim, H.L. (2018). An introduction to immunology and immunopathology. Allergy Asthma Clin. Immunol. 14(Suppl 2), 49.

Maywald, M., Wessels, I., and Rink, L. (2017). Zinc signals and immunity. Int. J. Mol. Sci. 18, 2222.

Mosser, D.M., Hamidzadeh, K., and Goncalves, R. (2021). Macrophages and the maintenance of homeostasis. Cell. Mol. Immunol. 18, 579-587.

Vincent, M.S., Gumperz, J.E., and Brenner, M.B. (2003). Understanding the function of CD1-restricted T cells. Nat. Immunol. 4, 517-523.

Vivier, E., Artis, D., Colonna, M., Diefenbach, A., Di Santo, J.P., Eberl, G., Koyasu, S., Locksley, R.M., McKenzie, A.N.J., Mebius, R.E., et al. (2018). Innate lymphoid cells: 10 years on. Cell 174, 1054-1066.

Waldmann, T.A. (2003). Immunotherapy: past, present and future. Nat. Med. 9, 269-277.

Yang, M., Liu, E., Tang, L., Lei, Y., Sun, X., Hu, J., Dong, H., Yang, S.M., Gao, M., and Tang, B. (2018). Emerging roles and regulation of MiT/TFE transcriptional factors. Cell Commun. Signal. 16, 31. 